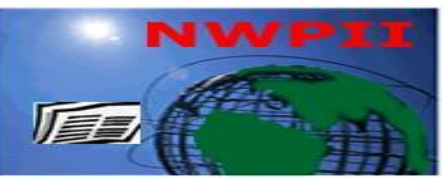

American Journal of Biomedical Sciences

ISSN: 1937-9080

nwpii.com/ajbms

\title{
Occurrence of Transfusion Transmissible Infections in Screened Blood with Rapid Diagnostic Kits in Comparison with ELISA Using Federal Medical Centre Owo as A Case Study
}

\author{
Joshua Seun FAPOHUNDA ${ }^{1}$, Adedeji David ATERE ${ }^{1,2^{*}}$, Aminat Abiola ADEBISI ${ }^{2}$ \\ ${ }^{1}$ Department of Medical Laboratory Science, University of Benin, Benin City, Edo State, Nigeria \\ ${ }^{2}$ Department of Medical Laboratory Science, Achievers University, Owo, Ondo State, Nigeria \\ Corresponding Author \\ A. D. Atere \\ Department of Medical Laboratory Science, \\ Achievers University, Owo \\ Nigeria \\ Email: ateread@gmail.com \\ Tel.: +2348039501172
}

Received:10 November 2018 ; | Revised:23 November 2018; | Accepted: 11 December 2018

\begin{abstract}
Transfusion medicine has a great public health importance worldwide because it has saved many lives. Every blood transfusion therefore carries a potential risk for transmissible diseases due to the lack of knowledge and unavailability of reference diagnostic techniques. The study is aimed at assessing the occurrence of transfusion transmissible infections in screened blood donors with rapid diagnostic kits in comparison with enzyme linked immunosorbent assay (ELISA) as a reference method. This is a cross sectional study and was carried out on fifty (50) apparently healthy individuals, whose ages ranged between 18 and 60 years, randomly recruited into this study from the Blood transfusion Unit, Federal Medical Centre, Owo. The blood of each donor was screened with rapid kits and ELISA for Hepatitis B virus (HBV), hepatitis $\mathrm{C}$ virus (HCV), Human immune deficiency virus (HIV) and syphilis. Of the 9 samples that initially tested positive for hepatitis B surface antigen (HBsAg) with rapid screening kit, 22.22\% tested negative with ELISA technique while out of 8 samples tested positive for VDRL, only one came out negative. The specificity and positive predictive value (PPV) for HBsAg and venereal disease research laboratory (VDRL) were $(95.3 \%, 77.8 \%)$ and $(97.7 \%, 87.5 \%)$ respectively while specificity and PPV for HIV and HCV were $100 \%$. It is thus necessary for Nigerian government to develop a safe blood donor screening strategy to reduce transfusion-transmitted infections by combining the use of less sensitive rapid screening with more sensitive techniques.
\end{abstract}

Keywords: Transfusion transmissible infections, Sensitivity, Specificity, Blood donors 


\section{Introduction}

Blood donation saves the lives of millions of people worldwide; however, the patients are at a potential risk of contracting transfusion-transmitted infections (TTIs), which in turn impose serious challenges to the medical providers for the availability of safe and affordable blood products ${ }^{[1]}$. According to the World health organization (WHO), safe blood is a universal right. A crucial requirement in the procurement of safe blood is to have a national program for donor selection, recruitment, retention, and education; this is said to minimize donations from donors who might transmit diseases to the recipients. Equally important is to evaluate the burden and risk factors for TTIs in the general population ${ }^{[2]}$. Hepatitis B virus (HBV), hepatitis C virus (HCV), Human immune deficiency virus (HIV) and syphilis are the most important agents causing transfusion transmitted infections (TTIs) and they constitute large health care burdens worldwide. Because of their latent nature prior to clinical presentation, their incidence rates are difficult to calculate ${ }^{[3]}$. Every blood transfusion therefore carries a potential risk for transmissible disease ${ }^{[4]}$. The accurate figures of TTIs in our population are still unknown due to the lack of understanding, un-availability of screening tests, limited access to health facilities and the unavailability of surveillance systems ${ }^{[5]}$. Furthermore, voluntary donors have been reported to be the safest group of donors because they usually have better health seeking behavior than the replacement blood donors and their intention is to donate blood to an unknown patient out of compassion $^{[1]}$.

Research have shown that Pre-donation testing of blood donors for Transfusion Transmissible Infections (TTIs) is done in most developing countries because substantial cost savings are made from resources, materials and man-hours which would have been spent to procure infected blood units. Simple rapid test kits used in pre-donation testing is not as sensitive as the Enzyme Linked Immuno-sorbent Assay (ELISA) method used in post-donation screening in a quality assured manner ${ }^{43}$. It is therefore worthwhile to study prevalence of transfusion transmissible infection in donor blood samples screened for HIV, Hepatitis B virus (HBV), hepatitis $\mathrm{C}$ virus (HCV) in Federal Medical Centre (FMC) Owo which uses rapid kit for blood transfusion screening.

\section{Materials and Methods}

\subsection{Study Design}

A total of 50 participants (aged 18-60years) were recruited for the study, after the ethical approval with registration number FMC/OW/380/VOL.LXIV/125 was obtained from the Federal Medical Centre (FMC) Ethics and Research Committee, Owo and also the informed consent was taken from each participant.

\subsection{Inclusion and Exclusion Criteria}

Donors of aged 18-60 years were recruited into the study irrespective of his/her blood groups. Pregnant women and breastfeeding mother were excluded from the study.

\subsection{Sample collection}

Eight (8) millilitres (mls) of venous blood was aseptically obtained from the antecubital fossa vein (with minimal stasis using pyrogen-free disposable needles and syringes) and dispensed. The samples dispensed into plain bottles was allowed to retract and serum was separated by centrifugation for 10 minutes at 4000rpm, into plain bottles and stored at $-20^{\circ} \mathrm{C}$ until time of analysis A third generation ELISA batch analyzer was used to test samples of corresponding donated units.

\subsection{Analytical Methods and Procedures}

The blood of each donor was screened with rapid kits for Hepatitis B virus (HBV), hepatitis C virus (HCV), Human immune deficiency virus (HIV) and syphilis. Thereafter, confirmation test was carried out on each test samples of corresponding donated units using Enzyme Linked Immunosorbent Assays (ELISA) technique (Genway Biotechnology, USA) following the manufacturers' instructions.

\subsection{Statistical Analysis}

The sensitivity and specificity calculations and estimation of negative and positive predictive values of the first/screening RDT were done by comparing its performance with ELISA (as a 
reference technique). Sensitivity of a test is defined as the ability to correctly identify the infected individuals; specificity as the ability to correctly identify the uninfected individuals; negative predictive value as the proportion of those with a negative test result who are uninfected and positive predictive value as the proportion of those with a positive test result who are actually infected. Sensitivity was calculated as true positives/(true positives + false negatives) $\times 100$; specificity as true negatives/(true negatives + false positives) $\times 100$; negative predictive value as true negatives/(true negatives + false negatives) $\times 100$ and positive predictive value as true positives/(true positives + false positives) $\times 100$.

\section{Results}

In this study, table 1 shows distribution of HIV, HBsAg, HCV and VDRL screening using both
ELISA and Rapid Kits tests. A total of fifty (50) selected subjects initially tested with HIV, HBsAg, HCV and VDRL and they were re-tested using ELISA technique. Of the 9 samples that initially tested positive for $\mathrm{HBsAg}$ with rapid screening kit, $2(22.22 \%)$ tested negative with ELISA technique while out of 8 samples tested positive for VDRL, only one came out negative. Similarly, comparison of results from rapid diagnostic test kits and ELISA were fully demonstrated in table 2 . The diagnostic validity performances of rapid kit tests were determined in comparison with ELISA method as a standard technique. The specificity and PPV for HBsAg and VDRL were $(95.3 \%, 77.8 \%)$ and $(97.7 \%, 87.5 \%)$ respectively while specificity and PPV for HIV and HCV were $100 \%$. Moreover, the sensitivity and NPV for HIV, HBsAg, HCV and VDRL were all $100 \%$ (table 3). Figure 1 shows graphical representation of PPV and NPV in percentage.

Table 1: Distribution of HIV, HBsAg, HCV and VDRL screening using both ELISA and Rapid Kits

\begin{tabular}{ccccc}
\hline & \multicolumn{2}{l}{ Results based on Rapid test Kit } & ELISA Result \\
& + Ve & - Ve & + Ve & -Ve \\
\hline N & 50 & 50 & 50 & 50 \\
HIV & 11 & 39 & 11 & 39 \\
HBsAg & 9 & 41 & 7 & 43 \\
HCV & 8 & 42 & 8 & 42 \\
VDRL & 8 & 42 & 7 & 43
\end{tabular}

Key: $+\mathrm{Ve}=$ Positive, $-\mathrm{Ve}=$ Negative, $\mathrm{N}=$ sample size, $\mathrm{HIV}=$ Human immune deficiency virus, HBsAg $=$ Hepatitis $\mathrm{B}$ surface antigen, $\mathrm{HCV}=$ Hepatitis $\mathrm{C}$ virus, $\mathrm{VDRL}=$ Venereal disease research laboratory

Table 2: Comparison of results from rapid diagnostic test kits and ELISA

\begin{tabular}{lccc}
\hline \multirow{2}{*}{$\begin{array}{l}\text { Rapid diagnostic } \\
\text { kit result }\end{array}$} & \multicolumn{2}{c}{ ELISA Test Result } & Total \\
\cline { 2 - 4 } HIV & $+\mathrm{Ve}$ & $-\mathrm{Ve}$ & \\
+Ve & 11 & 0 & 11 \\
-Ve & 0 & 39 & 39 \\
Total & 11 & 39 & 50 \\
\hline HBsAg & & & \\
+Ve & 7 & 2 & 9 \\
\hline -Ve & 0 & 41 & 41 \\
Total & 7 & 43 & 50 \\
HCV & & & 8 \\
\hline +Ve & 8 & 0 & 42 \\
-Ve & 0 & 42 & 50 \\
Total & 8 & 42 & 8 \\
VDRL & & & 43 \\
\hline +Ve & 7 & 1 & 50 \\
\hline -Ve & 0 & 42 & 43 \\
Total & 7 & 4 & \\
\hline
\end{tabular}


Key: $+\mathrm{Ve}=$ Positive, $-\mathrm{Ve}=$ Negative, HIV $=$ Human immune deficiency virus, $\mathrm{HBsAg}=$ Hepatitis B surface antigen, $\mathrm{HCV}=$ Hepatitis $\mathrm{C}$ virus, $\mathrm{VDRL}=$ Venereal disease research laboratory

Table 3: Sensitivity, Specificity, PPV and NPV of Rapid Kits used in HIV, HBV, HCV and VDRL tests

HIV HBV HCV VDRL

$\begin{array}{lrrrr}\text { Sensitivity (\%) } & 100 & 100 & 100 & 100 \\ \text { Specificity (\%) } & 100 & 95.3 & 100 & 97.7 \\ \text { PPV (\%) } & 100 & 77.8 & 100 & 87.5 \\ \text { NPV (\%) } & 100 & 100 & 100 & 100\end{array}$

Key: HIV = Human immune deficiency virus, HBsAg = Hepatitis B surface antigen, HCV = Hepatitis C virus, VDRL $=$ Venereal disease research laboratory, PPV $=$ Positive predictive value, NPV $=$ Negative predictive value

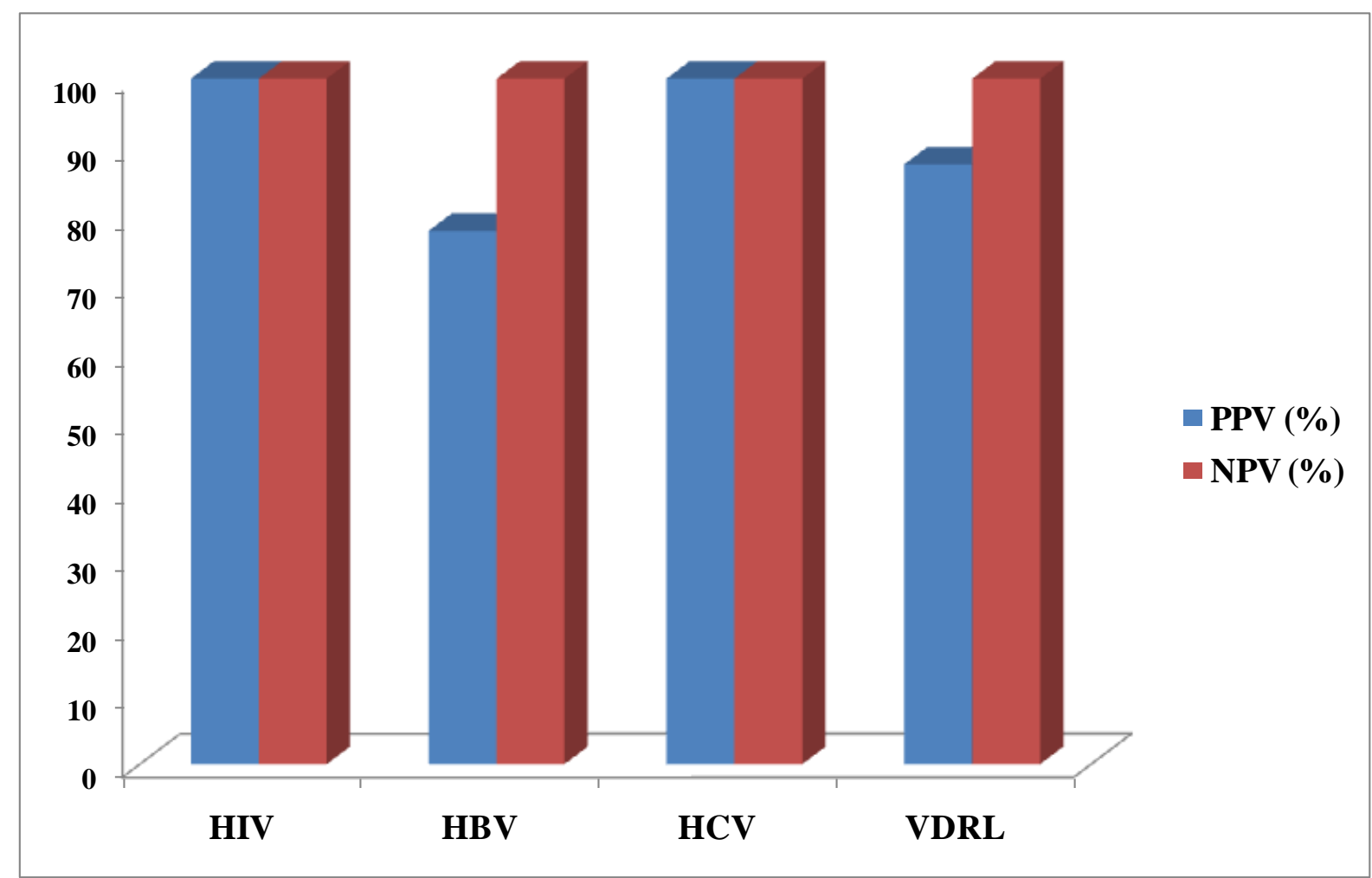

Figure 1: Positive predictive value (PPV) and Negative predictive value (NPV) of Kits and ELI used in HIV, HBV, HCV and VDRL tests in Percentage

\section{Discussion}

Transfusion-transmissible infections (TTI) like HIV, HBV, HCV and VDRL are one of the greatest threats to blood safety for transfusion recipients particularly in developing countries and pose a serious public health problem. ELISA and rapid kit tests (RKT) are both widely adopted immunological techniques for blood screening for TTI ${ }^{[6,12]}$. Improvement of immunoassay for the detection of viral infections, particularly HIV and hepatitis has always been predicted and thus it requires for early diagnosis of the disease and to rule out cross reaction issues.

In this study, we selected subjects initially tested with HIV, HBsAg, HCV and VDRL and they were re-tested using ELISA technique. Of the 9 samples that initially tested positive for $\mathrm{HBsAg}$ with rapid screening kit, $2(22.22 \%)$ tested negative with ELISA technique while out of 8 samples tested 
positive for VDRL, only one came out negative. Discrepancy between results obtained by the two techniques is not uncommon ${ }^{[7]}$. Some studies documented inferior diagnostic performance of RDTs in comparison with ELISA in India where both the modalities were used to screen healthy blood donors for HIV infection and the RKT used missed 17 of 30 samples confirmed reactive by ELISA ${ }^{[6]}$. This discordance might possibly be due to low antibody titres especially in recent infections where the levels may well be below the detection limit of RDTs but are picked up by the more sensitive enzyme immunoassay analysis. Whereas the discrepancy result obtained in this study might be due to cross reaction with other transfusiontransmissible infections with high antibody titre in the blood.

Furthermore, all samples that tested positive with HIV and HCV rapid screening kit also tested positive (100\% sensitivity) with the ELISA technique and those that tested negative with RKT also showed $100 \%$ specificity in comparison with the reference method. Even though we could not see any case of false negative result which had been reported by several researchers ${ }^{[6,7]}$ but we observed false positive results with the rapid diagnostic kit compared to ELISA in HBV and VDRL. This present study further shows that the rapid tests are inferior compared to ELISA and it is not the best options for blood screening.

Discordant results between 2 assays (positive with the rapid kit but negative with ELISA or vice vasa) for the diagnosis of an infectious disease can cause a huge challenge and have serious consequences among patients ${ }^{[8,9]}$. It can cause undue mental stress and tension. Kit evaluation is vital in determining the diagnostic kit of better performance. The ELISA test in this study showed a better performance (100\% of sensitivity, specificity, positive predictive value, and negative predictive value) value. Positive Predictive Value (PPV) is the ability of an assay to identify actual infected individuals among all persons giving a positive result with the kit being used. Negative predictive value is the ability of an assay to identify correctly the real non-infected individuals among persons giving a negative result with the kit being used. A good assay for an infectious agent like HIV and
HBV from a diagnostic point of view should be the one with high validity performance ${ }^{[10,11]}$.

In spite that the inferior diagnostic performance of RKT to ELISA had been reported, they still have some diagnostic merits. They still show high promising value of specificity and positive predictive value as it was observed in this study besides cost effectiveness. Rapid diagnostic tests (RDTs) can still be considered as alternative especially in indigenous area with poor power settings to maintain good supply chain. However ELISA and most recently nucleic acid testing (NAT) is advised to use along for blood transfusion screening in order to absolutely guarantee blood safety and to reduce potential risk of contracting transfusion-transmitted infections (TTIs), which may in turn impose serious challenges to the medical providers. The major challenges with ELISA and NAT is that they are more expensive, require use of instrumentation, time consuming and requires trained personnel. Our observation is probably, marred by the small sample size of this study, this indicates that there is a need for a large scale study to really delineate the real infection rate between the techniques.

\section{Conclusion and Recommendation}

There is increasing need for the Nigerian Government to develop a safe blood donor screening strategy for transfusion-transmitted infections (TTIs) by combining the use of less sensitive rapid screening techniques with more sensitive and sophisticated evidenced- based ELISA screening in order to ensure the safety of blood donation in the country.

\section{Competing interest}

The authors declare that they have no competing interests.

\section{Acknowledgement}

The authors appreciate the entire staff of Haematology and Blood Transfusion Unit of Federal Medical Centre, Owo and all the participants for their supports during this study. 


\section{References}

1 Arshad A, Borhany M, Anwar N, Naseer I, Ansari R, Boota S, Fatima N, Zaidi M, Shamsi $\mathrm{T}$. Prevalence of transfusion transmissible infections in blood donors of Pakistan. BMC Hematol 2016; 16: 27 DOI: 10.1186/s12878016-0068-2

2 Ahmad , G. An estimate of transfusiontransmitted infection prevalence in general populations. Hepatitis Mon 2011, 11(12):1002 -1003 .

3 Bhawani, Y., Rao, P.R. and Sudhakar, V. Seroprevalence of Transfusion Transmissible Infections among Blood Donors in a Tertiary Care hospital of Andhra Pradesh. Biological Medicine; 2010, (4):45-48.

4 Shittu AO, Olawumi HO, Adewuyi JO. Predonation screening of blood for transfusion transmissible infections: the gains and the pains - experience at a resource limited blood bank. Ghana Med J 2014; 48(3): 158-162 [PMID: 25709126 PMCID: PMC4335446]

5 Attaullah S, Khan S, Khan J. Trend of transfusion transmitted infections frequency in blood donors: provide a road map for its prevention and control. J Transl Med 2012; 10: 20 [PMID: 22293125 PMCID: PMC3286364 DOI: $\underline{10.1186 / 1479-5876-10-20]}$

6 Mehra B, Bhattar S, Bhalla P, Rawat D. Rapid Tests versus ELISA for Screening of HIV Infection: Our Experience from a Voluntary Counselling and Testing Facility of a Tertiary Care Centre in North India. ISRN AIDS 2014; 2014: 296840 [PMID: 25006527 PMCID: PMC4004236 DOI: $\underline{10.1155 / 2014 / 296840]}$
7 Iqbal HS, Solomon S, Murugavel KG, Solomon SS, Balakrishnan P. Evaluation and diagnostic usefulness of domestic and imported enzyme-linked immunosorbent assays for detection of human immunodeficiency virus type 1 antibody in India. Clin Diagn Lab Immunol 2005; 12(12): 1425-1428 DOI: $10.1128 / C D L I .12 .12 .1425-$ $\underline{1428.2005}$

8 Khan, J.K., Lone, D.S., Hameed, A., Munim, M.R., Satti, H.S. and Munir, M. Evaluation of the performance of two rapid immunochromatographic test for the detection of hepatitis B surface antigen and anti-HCV antibodies using ELISA tested samples. $\boldsymbol{S p} \boldsymbol{E} \boldsymbol{d}$ Ann; 2010, 16: 84-87.

9 Maity S, Nandi S, Biswas S, Sadhukhan SK, Saha MK. Performance and diagnostic usefulness of commercially available enzyme linked immunosorbent assay and rapid kits for detection of HIV, HBV and $\mathrm{HCV}$ in India. Virol J 2012; 9: 290 DOI: 10.1186/1743422X-9-290

10 World Health Organization (WHO). Status of blood safety in the WHO African Region. Report of the 2007 Survey WHO Regional Office for Africa, Brazzaville.

11 Zameer, M., Shahzad, F., Shafi, F., Ali, K.H. and Saeed, U. et al. Transfusion Transmissible Infections among Healthy Blood Donors. Pak Armed Forces Medical Journal; 2017, 67(1):131-136.

12 Fasakin, K.A. and Ajayi, O.D. Hepatitis B Vaccination Outcomes and Broad Spectrum of Hepatitis B Infections in Nigeria: An Evidence-Based Picture. Am. J. Biomed. Sci. 2017, 9(4): 211-224. doi:10.5099/aj17040021 